\title{
The role of azacitidine in the management of myelodysplastic syndromes (MDS)
}

This article was published in the following Dove Press journal:

Cancer Management and Research

8 October 2009

Number of times this article has been viewed

\section{KS Götze \\ C Müller-Thomas \\ C Peschel}

Department of Medicine, Hematology/ Oncology, Technische Universität München, Munich, Germany
Correspondence: Katharina S Götze III Dept of Medicine, Hematology/ Oncology, Technische Universität München, Ismaningerstr 22, D-81675 Munich, Germany

Tel +4989 4I4056I8

Fax +49894140 4879

Email k.goetze@Irz.tum.de
Abstract: Myelodysplastic syndromes (MDS) are a group of common bone marrow disorders characterized by ineffective hematopoiesis, peripheral cytopenias, and a propensity for transformation to acute myeloid leukemia (AML). For many years, the main treatment option for MDS was best supportive care which alleviates symptoms but has no effect on the natural course of the disease. The recent approval of the demethylating agent azacitidine represents a significant advance in the treatment of MDS. The results of two randomized trials with azacitidine have shown an overall response rate between $40 \%$ and $60 \%$, an improved quality of life, a reduced risk of transformation to AML and a definite survival advantage compared to best supportive care or low-dose chemotherapy. Current data on azacitidine and its place in the treatment of MDS are reviewed.

Keywords: myelodysplastic syndromes, MDS, azacitidine, epigenetic therapy, demethyltransferase inhibitor

\section{Introduction}

Myelodysplastic syndromes (MDS) encompass a group of hematopoietic stem cell malignancies characterized by ineffective hematopoiesis, peripheral cytopenias, and a propensity for transformation into acute myeloid leukemia (AML). The disease afflicts approximately 5 in every 100,000 individuals in the general population but incidence rises sharply with age, making MDS the most common hematologic cancer in the age group over seventy. ${ }^{1-3}$ With the exception of allogeneic stem cell transplantation, MDS remains incurable and most patients eventually die from their disease, most often due to infectious complications or transformation to AML. ${ }^{4}$ Thus, improving outcomes for patients with MDS remains an important goal in clinical hematology.

The prognosis for individual MDS patients varies greatly and ranges from relatively indolent disease with longer life expectancy to more aggressive courses with rapid progression to overt leukemia. Prognosis depends on several factors, including the presence of chromosomal abnormalities, number of cytopenias, and percentage of bone marrow blasts as well as transfusion dependence. The most commonly employed score for prognostication in MDS is the risk-based International Prognostic Scoring System (IPSS). ${ }^{4}$ By combining blast percentage, karyotype, and number of cytopenias the IPSS provides a reliable estimate of survival and risk of transformation to AML for patients with MDS, ${ }^{4}$ although the prognostic importance of chromosomal abnormalities may be underestimated in this score. Using the IPSS, 4 distinctive risk groups can be identified which differ in terms of overall survival and AML evolution (low, intermediate-1, intermediate-2, and high risk). The IPSS can be combined 
with French-American-British (FAB) or World Health Organization morphologic criteria and additional variables such as the requirement for regular transfusions and serum LDH levels to further improve discrimination. ${ }^{5-7}$ MDS patients with a low or intermediate-1 IPSS score have a fairly favorable median survival of 5.7 and 3.5 years, respectively. In contrast, patients with higher-risk MDS (ie, Int-2 or high IPSS score) fare significantly worse with a median survival of 1.2 or 0.4 years, respectively, and a high risk of progression to AML. ${ }^{4}$ MDS patients with lower-risk IPSS scores who are transfusion dependent also have a poor expected median overall survival.

In general, management of MDS depends on the IPSS score and transfusion requirement of the individual patient as well as age and comorbidities. The latter factors are especially relevant in the context of evaluation for allogeneic stem cell transplantation. Historically, the major goal of therapy for MDS patients with lower-risk disease (ie, IPSS low or Int-1 category) has been alleviation of symptoms and improvement in quality of life through correction of existing cytopenias, although prolonging survival is desirable as well. In contrast, the very poor prognosis of higher-risk MDS patients (ie, IPSS Int-2 or high-risk categories) makes extension of survival the major treatment objective in this subgroup. For these reasons, it has been common practice to stratify MDS patients according to IPSS risk into a lower-risk and a higher-risk group, respectively. However, as diagnosis of MDS subgroups becomes more refined through utilization of new molecular techniques and at the same time more effective therapies become available, the current stratification of risk groups as well as the therapeutic goals for these groups may have to be adapted. This review aims to summarize currently available therapeutic options for MDS patients with a particular focus on the role of the demethylating agent azacitidine.

\section{Existing treatment options in MDS}

For many years, the main treatment option for MDS was best supportive care (BSC) which aims to alleviate the negative effects of cytopenia and to increase quality of life. Even with the emergence of novel drugs, BSC remains the backbone of MDS therapy for all patients. This includes red blood cell transfusions to alleviate symptoms of anemia, platelet transfusions to decrease risk of hemorrhage in thrombocytopenic patients, adequate iron chelation therapy to reduce hemosiderosis, and control of infections by the use of antibiotics and granulocyte-colony stimulating factor (G-CSF) as appropriate.
In patients with lower-risk MDS, specific therapy beyond BSC should be considered if symptomatic cytopenia is present. For symptomatic anemia, treatment with erythropoiesis-stimulating agents (ESAs) such as erythropoietin or darbepoietin with or without the addition of G-CSF can be attempted in patients with a favorable response profile (ie, modest transfusion requirement and low erythropoietin serum levels). ${ }^{8,9}$ Patients suffering from isolated severe neutropenia and recurrent infections may benefit from use of G-CSF. Symptomatic thrombocytopenia is presently managed with platelet transfusions although thrombopoietin analogues may represent a therapeutic option in the future. For transfusion-dependent lower-risk patients with chromosome $5 \mathrm{q}$ deletion $(\operatorname{del}(5 \mathrm{q}))$, the immunomodulatory agent lenalidomide is considered the primary treatment of choice as it has shown very high remission rates with achievement of transfusion independence. ${ }^{10,11}$ Lenalidomide may also be useful in the treatment of lower-risk MDS patients without $\operatorname{del}(5 q)$ who do not respond to ESAs. ${ }^{12}$ Immunosuppressive therapy is an option for a very small subgroup of lower-risk MDS patients, especially younger patients with hypocellular bone marrow or HLA-DR15+ subtype and a low transfusion burden. ${ }^{13,14}$ While all of these treatment options may lead to improvement in blood values and an increased quality of life in patients with lower-risk MDS, a true survival benefit has so far been shown retrospectively for the use of erythropoietin + G-CSF only in the subgroup of patients with a low transfusion requirement. ${ }^{15,16}$

For higher-risk MDS patients, therapeutic options have been even more limited. Until recently there has been an absence of satisfactory treatment options bridging the gap between $\mathrm{BSC}$ on the one end and allogeneic stem cell transplantation (SCT) on the other end of the treatment spectrum. The only proven curative approach for MDS is allogeneic SCT, ${ }^{17-19}$ while other less intensive treatment strategies including non-intensive chemotherapy, immunomodulatory agents or immunosuppressive therapy have not been able to change the natural course of the disease in higher-risk MDS patients. ${ }^{20}$ Allogeneic stem cell transplantation has been shown to confer an overall survival benefit to patients with MDS with long-term event-free survival rates of $28 \%$ to $60 \%$, depending on the IPSS score, transfusion dependence and comorbidities..$^{20-23}$ The most common approach is standard myeloablative conditioning, although reduced intensity conditioning is now also used more often, allowing older patients to undergo this procedure. ${ }^{18,19,24,25}$ Transplant-related mortality is in the range of $30 \%$ to $40 \%$ in most studies and is a major cause of death, as is relapse 
after transplantation. ${ }^{26,27}$ Given these limitations as well as the prevalence of patients above the age of 70 years with considerable comorbidities, allogeneic SCT is not a realistic option for most patients with higher-risk MDS.

Demethylating agents such as azacitidine (Vidaza ${ }^{\circledR}$; Celgene Corp., Summit, NJ, USA) and 5-aza-deoxycitidine (decitabine, Dacogen ${ }^{\circledR}$; Eisai Inc., Woodcliff Lake, NY, USA) have recently emerged as promising drugs for the treatment of MDS. With proven efficacy in terms of remission rates as well as increased progression-free survival for all MDS subtypes and improved overall survival in higherrisk MDS, azacitidine now represents the best alternative therapeutic option for those higher-risk MDS patients not eligible for allogeneic SCT. It may also be considered in transfusion dependent lower-risk MDS patients who have failed alternative therapies. Azacitidine was approved for the treatment of patients with MDS of all subtypes in 2004 by the US Food and Drug Administration (FDA) and for higherrisk MDS, CMML and AML with $20 \%$ to $30 \%$ blasts and multi-lineage dysplasia by the European Medicines Agency (EMEA) in 2009.

\section{DNA methylation and epigenetic therapy}

Methylation of DNA is an epigenetic modification the cell uses to control certain processes such as gene transcription. In normal cells, much of the genome is methylated. DNA methylation patterns are specific for different tissues and are conserved through cell divisions, allowing the expression of the particular set of genes necessary for that cell type and inhibiting unchecked copying of repeat sequences. ${ }^{28}$ Epigenetic modification of DNA is achieved by methylation of cytosine residues within $\mathrm{CpG}$ islands. ${ }^{29}$ These $\mathrm{CpG}$ islands located in the proximal gene promoter regions are short 0.5 to $4 \mathrm{~kb}$ length regions rich in $\mathrm{CpG}$ dinucleotides. They are generally unmethylated in normal cells. Methylation of $\mathrm{CpG}$ islands changes the conformation of the chromatin, leading to inhibition of gene transcription and thus, gene silencing. ${ }^{29}$ In cancerous cells, DNA methylation is often lost or aberrantly increased, leading to abnormal gene expression. In particular, hypermethylation of $\mathrm{CpG}$ islands of tumor-suppressor genes is a common alteration in cancer cells. $^{28,29}$ Methylation of DNA is accomplished by DNA methyltransferases (DNMTs) and elevated levels of DNMTs are commonly observed in human tumors. ${ }^{30}$

Among the tumor suppressor genes which have been shown to be epigenetically silenced in MDS are $\mathrm{p} 15^{\text {ink4b }}$, which encodes a cyclin-dependent kinase inhibitor and
CDH1, which encodes the adhesion molecule E-cadherin. ${ }^{31,32}$ The presence of $\mathrm{p} 15^{\mathrm{ink} 4 \mathrm{~b}}$ methylation is more frequent in higher-risk MDS and has been shown to correlate with disease progression. ${ }^{33-35}$ Similarly, methylation of CDH1 has been shown to have a negative prognostic impact in MDS, with inferior remission rates in response to chemotherapy in patients showing hypermethylation of this gene. ${ }^{36}$

The demethylating agents azacitidine and decitabine have been shown to act as DNMT inhibitors. Incorporation of azacitidine or decitabine into DNA results in a dose- and time-dependent inhibition of DNA methyltransferase activity by irreversible binding to the enzyme, thus preventing methylation of newly synthesized DNA. This allows a reversal of epigenetically silenced genes, restoring normal growth control and differentiation to impaired hematopoietic cells. ${ }^{29,37}$ Accordingly, restoration of $\mathrm{p} 15^{\mathrm{ink} 4 \mathrm{~b}}$ expression through hypomethylation has been demonstrated in bone marrow cells from MDS patients treated with decitabine. ${ }^{38}$ However, a second study failed to show a correlation between DNA demethylation of $\mathrm{p} 15^{\mathrm{ink} 4 \mathrm{~b}}$ and clinical response to treatment with azacitidine, although lower baseline methylation was noted in responding patients. ${ }^{39}$

In addition to their demethylating properties, azacitidine as well as decitabine have been shown to induce direct cytotoxicity to cancer cells. However, the complete mode of action of either of these drugs has not yet been fully delineated and is the subject of ongoing research.

\section{Pharmacology of azacitidine and decitabine}

Azacitidine (5-azacitidine) is an analogue of the naturally occurring pyrimidine nucleoside cytidine. Azacitidine differs from cytidine in containing a nitrogen atom at the 5 position of the heterocyclic ring instead of a carbon atom (Figure 1). The chemical name of azacitidine is 4-amino-1- $\beta$ D-ribofuranosyl-s-triazin-2(1H)-one, the empirical formula of azacitidine is $\mathrm{C}_{8} \mathrm{H}_{12} \mathrm{~N}_{4} \mathrm{O}_{5}$ and the molecular weight is $244 .{ }^{40}$ Azacitidine is a prodrug of 5-aza-deoxycitidine (decitabine). The chemical name of decitabine is 4-amino-1-(2-deoxy- $\beta$ D-erythro-pentofuranosyl)-1,3,5-triazin-2(1H)-one. It has the molecular formula $\mathrm{C}_{8} \mathrm{H}_{12} \mathrm{~N}_{4} \mathrm{O}_{4}$ and the molecular weight of 228.21 .

Upon uptake into proliferating cells, azacitidine is phosphorylated to azacitidine monophosphate and then to diphosphate and triphosphate. The triphosphate metabolite is incorporated into RNA, leading to disruption of RNA metabolism and consecutively to inhibition of protein synthesis. The diphosphate metabolite is further reduced to a deoxynucleotide 


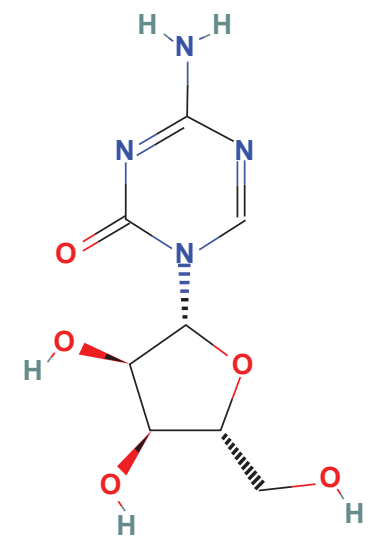

5-azacitidine

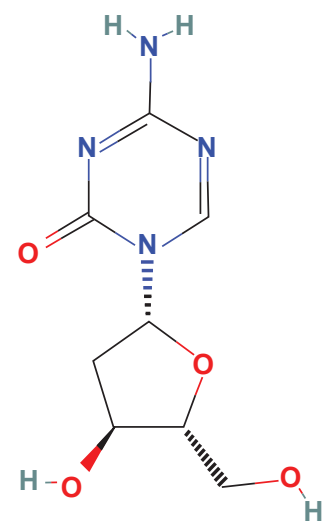

\section{5-aza-2'-deoxycitidine}

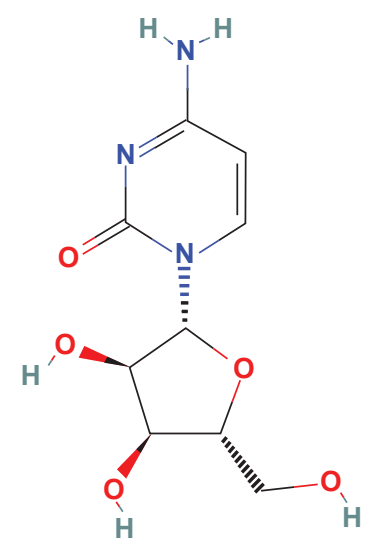

cytidine

Figure I Molecular structure of azacitidine, decitabine, and cytidine.

form (5-aza-deoxycitidine, decitabine) and then incorporated into DNA where it irreversibly binds and inhibits DNA methyltransferase. ${ }^{41,42}$ Decitabine is directly incorporated into DNA after activation by deoxycitidine kinase. ${ }^{43}$

Azacitidine is chemically unstable and therefore manufactured as a lyophilized powder. Upon reconstitution in sterile water azacitidine is stable for up to 8 hours if refrigerated but must otherwise be administered within 1 hour of preparation. ${ }^{40}$ After administration by subcutaneous or intravenous route, azacitidine is rapidly absorbed within 10 to 30 minutes. The plasma half-life is 20 and $40 \mathrm{~min}-$ utes for intravenous and subcutaneous administration, respectively. ${ }^{44}$ Azacitidine is widely distributed in tissues and eliminated primarily by urinary excretion. No studies on interactions between azacitidine and other drugs have been conducted.

Decitabine is also rapidly absorbed within 35 minutes and widely distributed in tissues. Decitabine crosses the blood-brain barrier achieving $27 \%$ to $58 \%$ of the plasma concentrations after continuous infusion. ${ }^{45}$ Decitabine is inactivated through its major elimination pathway by liver cytidine deaminase. ${ }^{42}$

\section{Efficacy studies with azacitidine}

Clinical data on the efficacy of azacitidine in the treatment of MDS are available from 2 phase II and 2 randomized phase III clinical trials. ${ }^{46-48}$ A total of 191 MDS patients were enrolled into the first phase III CALGB 9221 trial. ${ }^{46}$ Patients were randomized to receive $\mathrm{BSC}$ or azacitidine by subcutaneous injection at a dose of $75 \mathrm{mg} / \mathrm{m}^{2}$ on 7 consecutive days every 28 days. All subtypes of MDS could be enrolled. However, patients with MDS subtypes RA/RARS were included only if they had severe thrombocytopenia or neutropenia or transfusion-dependent anemia. After a minimum of 4 months of supportive care, patients whose disease progressed were permitted to cross over to azacitidine treatment. A total of 99 patients were primarily treated with azacitidine and an additional 49 patients (55\% of patients in the observation arm) crossed over to azacitidine after 4 months. Approximately half of the total patient population had higher-risk MDS as determined by IPSS criteria. The primary endpoint of the study was overall response rate.

According to the International Working Group (IWG) criteria for assessing responses in $\mathrm{MDS},{ }^{49,50}$ the overall response rate in the azacitidine arm was $47 \%$ (Table 1). ${ }^{47}$ Responses to azacitidine were observed in all FAB subtypes. The majority of patients showed hematologic improvement while $11 \%$ achieved either a complete response (CR) or partial response (PR). There were no complete or partial remissions in the observation arm but $17 \%$ of patients also showed hematologic improvement under BSC. Patients treated with azacitidine showed a significant delay in progression to AML compared to the BSC arm (21 vs 13 months, $P=0.007$ ). Improved survival by azacitidine treatment could not be established due to 
the crossover design and cohort size. However, an absolute difference in survival of 6 months in favor of azacitidine (20 months vs 14 months for BSC) was documented, suggesting a possible survival benefit.

Among the 99 patients randomized to azacitidine, 66\% were transfusion dependent at baseline. Of these, $45 \%$ had an elimination of all transfusions while another $9 \%$ had a reduction in transfusions by $50 \%$. In addition, lineage responses for platelets and white blood cells occurred in $47 \%$ and $40 \%$, respectively, among the patients treated with azacitidine. The median duration of transfusion independence was 9 months.

To definitely ascertain whether treatment with azacitidine conferred a survival benefit to MDS patients, the large randomized phase III AZA-001 trial was initiated. This trial with the primary endpoint overall survival included 358 patients with only higher-risk MDS (Int-2 or high-risk according to IPSS).$^{48}$ Patients were randomized to receive either azacitidine (179 patients) at a dose of $75 \mathrm{mg} / \mathrm{m}^{2}$ for 7 days or 1 of 3 conventional care regimens (CCR). The choice of conventional care regimens included BSC (105 patients), low-dose cytarabine (49 patients) or intensive chemotherapy (25 patients) as selected by investigators before randomization. This trial showed a remission rate $(\mathrm{CR}+\mathrm{PR})$ of $29 \%$ for azacitidine compared to $12 \%$ for CCR $(P<0.0001)$ and a hematological improvement rate of $49 \%$ for azacitidine compared to $29 \%$ for CCR $(P<0.0001)$ (Table 2). Transfusion independence was achieved in $45 \%$ of patients receiving azacitidine who were dependent on red blood cell transfusions at baseline. More importantly, a significantly prolonged median

Table I Response to azacitidine (AZA) compared to best supportive care (BSC) in the randomized CALGB 9221 trial according to InternationalWorking Group (IWG) response criteria for MDS $^{47}$

\begin{tabular}{llll}
\hline Response & $\begin{array}{l}\text { AZA } \\
(\mathbf{9 9} \text { pts) }\end{array}$ & $\begin{array}{l}\text { BSC } \\
\mathbf{( 9 2} \text { pts) }\end{array}$ & P value \\
\hline CR (\%) & 10 & 0 & \\
PR (\%) & 1 & 0 & \\
HI (\%) & 36 & 17 & $<0.001$ \\
Total response rate (\%) & 47 & 17 & 0.007 \\
Time to AML or death & 21 & 13 & \\
(months) & & & 0.001 \\
$\begin{array}{l}\text { Transformation to AML } \\
\text { as Ist event }\end{array}$ & 15 & 38 & \\
\hline
\end{tabular}

Abbreviations: $\mathrm{AML}$, acute myeloid leukemia; $\mathrm{CR}$, complete remission; $\mathrm{PR}$, partial remission; HI, hematologic improvement. overall survival for the azacitidine group compared to the CCR group was demonstrated (24.5 vs 15.0 months, respectively, $P=0.0001$ ) (Figure 2 ). In addition, time to AML progression as a secondary study endpoint was also significantly increased in the azacitidine arm (17.8 vs 11.5 months, $P<0.0001)$.

Thus, treatment with azacitidine prolongs overalls survival and lowers the risk of progression to AML in patients with higher-risk MDS. The absolute survival benefit for patients treated with azacitidine compared to conventional care was 9.4 months in the AZA-001 trial. The overall survival advantage at 2 years was 2 -fold higher in the azacitidine arm than the CCR arm (azacitidine: 51\%; CCR: 26\%; $P<0.0001)$. The results of the randomized AZA-001 trial make azacitidine the first specific therapy to show a definite survival benefit for MDS patients in a prospective randomized study.

\section{Comparison to decitabine}

In contrast to results of the AZA-001 study for azacitidine, clinical trials for decitabine have thus far not been able to show an overall survival benefit in patients with MDS. Initial phase II trials in Europe using decitabine at a total dose of $225 \mathrm{mg} / \mathrm{m}^{2}$ as a continuous infusion over 72 hours or at a total dose of $135 \mathrm{mg} / \mathrm{m}^{2}$ using a 3-day intravenous administration over 4 hours 3 times a day showed a favorable overall response rate of $49 \%$ to $54 \%$, comparable to that of azacitidine. ${ }^{51,52}$ In a randomized phase III trial testing decitabine in a schedule of $15 \mathrm{mg} / \mathrm{m}^{2}$ for a total of nine doses over 72 hours compared to BSC, the overall response rate was $30 \%{ }^{53}$ Patients with IPSS high-risk

Table 2 Secondary endpoints in the AZA-00I trial comparing azacitidine (AZA) to conventional care regimens (CCR)

\begin{tabular}{llll}
\hline & AZA & CCR & P value \\
\hline $\begin{array}{l}\text { Remission rate } \\
(\mathrm{CR}+\mathrm{PR}, \%)\end{array}$ & 29 & 12 & $0.000 \mathrm{I}$ \\
$\begin{array}{l}\text { Time to AML } \\
\text { transformation } \\
\text { (months) }\end{array}$ & 26.1 & 12.4 & 0.004 \\
$\begin{array}{l}\text { Transfusion } \\
\text { independence (\%) }\end{array}$ & 45 & II & $<0.000$ I \\
$\begin{array}{l}\text { Rate of infections } \\
\text { requiring antibiotics }\end{array}$ & $\begin{array}{l}\text { Reduced by 33\% in AZA } \\
\text { arm vs CCR arm }\end{array}$ & \\
\hline
\end{tabular}

Reprinted from Lancet Oncol, I 0, Fenaux P, Mufti GJ, Hellstrom-Lindberg E, et al. Efficacy of azacitidine compared with that of conventional care regimens in the treatment of higher-risk myelodysplastic syndromes: a randomised, open-label, phase III study, 223-232, ${ }^{48}$ Copyright (C) 2009, with permission from Elsevier.

Abbreviations: AML, acute myeloid leukemia; CR, complete remission; PR, partial remission. 


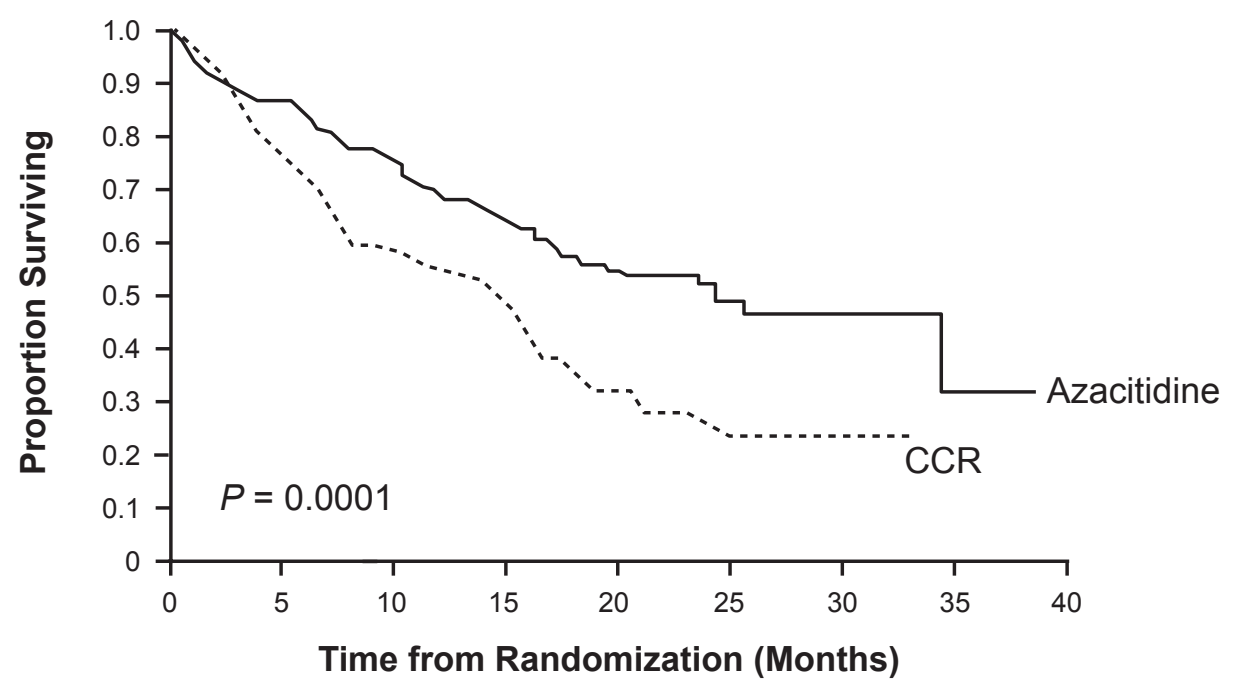

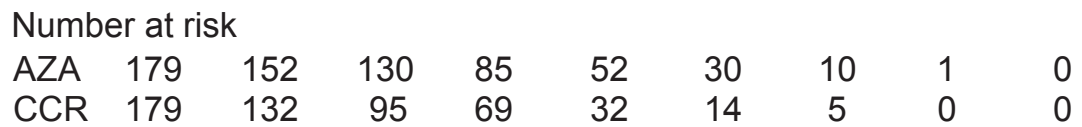

Figure 2 Overall survival in the AZA-00I trial by intention-to-treat analysis.

Reprinted from Lancet Oncol, I0, Fenaux P, Mufti GJ, Hellstrom-Lindberg E, et al. Efficacy of azacitidine compared with that of conventional care regimens in the treatment of higher-risk myelodysplastic syndromes: a randomised, open-label, phase III study, 223-232,48 Copyright @ 2009, with permission from Elsevier.

Abbreviation: CCR, conventional care regimens.

MDS receiving decitabine had a prolonged median time to progression to AML or death compared to patients receiving BSC but overall survival was not improved. ${ }^{53}$ Other clinical benefits of decitabine treatment were a reduction in growth factor and transfusion requirement and an increased quality of life. ${ }^{53}$ Recently published results of a schedule which can be administered in the outpatient setting using $20 \mathrm{mg} / \mathrm{m}^{2}$ decitabine as intravenous infusion for 5 consecutive days yielded comparable clinical efficacy. ${ }^{54}$ The preliminary report of the recently completed randomized EORTC clinical trial comparing decitabine to BSC has also shown a benefit for progression-free survival but not for overall survival. ${ }^{55}$

The reasons for the discrepancy between azacitidine and decitabine in terms of survival benefit are unclear. One of the major differences in trial design was the number of treatment cycles administered. While treatment was continued until progression in the AZA-001 trial and a median of 9 cycles were administered, decitabine was administered for a maximum of only 6 cycles in the EORTC trial. Continuation of treatment therefore seems to be important to achieve maximum benefit. In addition, patient selection varied between the two trials. While the AZA-001 study enrolled patients on the basis of IPSS score (Int-2 and high-risk) and included patients with up to $30 \%$ bone marrow blasts, the EORTC study required patients to have either $10 \%$ to $20 \%$ blasts or unfavorable cytogenetics. The different dosing regimens as well as the length of each cycle of the two drugs could also play a role. ${ }^{56}$ The more direct metabolic activation pathway to DNA incorporation of decitabine compared to the primarily RNA incorporation of azacitidine has been suggested to make decitabine a more potent compound than azacitidine. However, inhibition of DNA methyltransferase and hypomethylation may not be the only relevant mechanism for these drugs to elicit their clinical effect. Incorporation into RNA by azacitidine may also contribute to its anti-tumor effects. Further investigation in this area is necessary to understand the differences between these two demethylating agents. In addition, direct comparisons between azacitidine and decitabine are needed before conclusions about the relative efficacy of these two agents can be made.

\section{Cytogenetics and response to azacitidine}

Treatment with azacitidine also confers benefit in higher-risk MDS patients with cytogenetic abnormalities. There are various recurring cytogenetic abnormalities frequently associated with MDS which confer a poor prognosis. This is especially true for alterations involving chromosome 7 , either in the form of deletion (del (7q)) or absence (monosomy 7). ${ }^{5}$ Complex aberrant karyotypes are also associated with inferior 
outcome and are considered high risk whereas deletions of chromosome 20 (del(20q)) or isolated deletion of chromosome $5(\operatorname{del}(5 q))$ have a good prognosis. ${ }^{4,57}$ MDS patients with chromosome 7 abnormalities do not respond to conventional chemotherapy, making them poor candidates for intensive therapy if they are too old or frail to proceed to allogeneic stem cell transplantation. ${ }^{58} \mathrm{~A}$ high rate of remission in MDS patients with chromosome 7 abnormalities treated with azacitidine was first observed in a smaller uncontrolled study. ${ }^{39}$ In the randomized AZA-001 trial, treatment with azacitidine was shown to be beneficial for all cytogenetic subgroups of MDS including the group of patients with complex aberrations or chromosome 7 abnormalities (Table 3). ${ }^{59}$ Overall survival in the subgroup of patients with chromosome 7 abnormalities was 13.1 months for the azacitidine arm vs only 4.6 months in the conventional care arm $(P=0.003) .{ }^{60}$ Thus, azacitidine extends survival for this subgroup of MDS patients with a very poor prognosis, for whom no real therapeutic options existed in the past. Patients with complex aberrant cytogenetics treated with azacitidine showed a similar survival benefit. Of note, decitabine has also been shown to be very active in patients with chromosome 7 abnormalities. ${ }^{61}$ These favorable results suggest azacitidine or decitabine should be considered as therapy of choice in patients with chromosome 7 abnormalities or complex karyotypes ineligible for allogeneic SCT. MDS patients with intermediate or good prognostic karyotypes also fared better under therapy with azacitidine than with CCR. The median overall survival for the azacitidine arm was not reached in the good risk subgroup and was 26.3 months in the intermediate risk subgroup compared to 17 months for both cytogenetic groups in the CCR arm $(P=0.04$ and 0.02 , respectively $){ }^{48}$

\section{Safety and tolerability of azacitidine}

Since azacitidine is primarily excreted by the kidneys, the risk of toxicity is expected to be greater in patients with impaired renal function, although no formal studies have been performed. Likewise, the safety and pharmacokinetics of azacitidine have not been investigated in patients with evidence of hepatic impairment. It is therefore recommended that patients with renal or hepatic impairment be monitored closely. Azacitidine is contraindicated in patients with advanced malignant hepatic tumors. Azacitidine is potentially teratogenic. While this is not a concern for most elderly patients with MDS, women of childbearing potential should avoid becoming pregnant during therapy with azacitidine, and men should avoid fathering a child.

The most common adverse event occurring during treatment with azacitidine is myelosuppression. Since cytopenias are inherent to MDS, evaluation of treatment-induced myelosuppression is difficult to assess. However, low baseline blood cell counts further decreased in patients treated with azacitidine. Accordingly, the most common reasons for treatment discontinuation, dose reduction or interruption were leukocytopenia, neutropenia and thrombocytopenia. In the CALGB 9221 trial, grade 3-4 leukocytopenia occurred in $59 \%$, granulocytopenia in $81 \%$ and thrombocytopenia in $70 \%$ of patients receiving azacitidine. ${ }^{46}$ Infection thought to be related to treatment was reported in $20 \%$ of patients. The highest rate of adverse events was reported during the first 2 cycles of therapy and decreased in subsequent cycles. The proportion of patients with adverse events did not increase with patient age. There was 1 treatment-related death $(<1 \%)$ in the CALGB 9221 trial and 4 treatment-related deaths $(2 \%)$ in the AZA-001 trial. In the AZA-001 trial, rates of grade 3-4 toxicity were $91 \%$ for neutropenia, $85 \%$ for thrombocytopenia and $57 \%$ for anemia. ${ }^{48}$ Five percent of patients receiving azacitidine discontinued treatment in the AZA-001 trial before study completion owing to hematological adverse events.

Non-hematological adverse events were most commonly either gastrointestinal or associated with administration. Injection site reactions in the form of localized erythema or bruising occur in almost all patients with subcutaneous application of azacitidine. Generally, these reactions are

Table 3 Median overall survival in AZA-00I trial according to cytogenetic risk

\begin{tabular}{|c|c|c|c|c|c|}
\hline \multirow[t]{2}{*}{ Cytogenetic risk group } & \multirow[t]{2}{*}{$\%$ pts $(\mathbf{n} / \mathbf{N})$} & \multicolumn{2}{|c|}{$\begin{array}{l}\text { Median overall survival } \\
\text { (months) }\end{array}$} & \multirow[t]{2}{*}{ HR (95\% Cl) } & \multirow[t]{2}{*}{$\begin{array}{l}P \text { value } \\
\text { log rank }\end{array}$} \\
\hline & & AZA & CCR & & \\
\hline Good (normal, del(5q), del(20q) & $46(166 / 358)$ & Not reached & 17.1 & $0.61(0.39-0.96)$ & 0.030 \\
\hline Intermediate (other) & $21(76 / 358)$ & 26.3 & 17.0 & $0.43(0.21-0.88)$ & 0.017 \\
\hline Poor (complex, -7) & $28(100 / 358)$ & 17.2 & 6.0 & $0.52(0.32-0.87)$ & 0.011 \\
\hline
\end{tabular}

Reprinted from Lancet Oncol, I0, Fenaux P, Mufti GJ, Hellstrom-Lindberg E, et al. Efficacy of azacitidine compared with that of conventional care regimens in the treatment of higher-risk myelodysplastic syndromes: a randomised, open-label, phase III study, 223-232, ${ }^{48}$ Copyright (C 2009, with permission from Elsevier.

Abbreviations: AZA, azacitidine; $\mathrm{CCR}$, conventional care regimens; $\mathrm{Cl}$, confidence interval; $\mathrm{HR}$, hazard ratio. 
transient and do not require specific therapy. Common gastrointestinal side effects include nausea, vomiting, diarrhea, constipation and anorexia. Table 4 summarizes the most common side effects during azacitidine treatment and their management. Other reported less common adverse events were arthralgia, cough, headache, weakness, dizziness and insomnia. Liver function abnormalities occurred in patients with intercurrent illnesses and more severe abnormalities were noted in patients with liver cirrhosis. ${ }^{44}$ Adverse events occurred at about the same frequency in males and females and in all age groups. ${ }^{40}$ Treatment of older patients $\geq 75$ years was not associated with increased toxicity. ${ }^{62}$

\section{Dosing of azacitidine and duration of therapy}

The standard dosing regimen for azacitidine employed in the CALGB 9221 as well as the AZA-001 trial is $75 \mathrm{mg} / \mathrm{m}^{2}$ for 7 consecutive days repeated every 28 days (AZA-7). Patients should be premedicated for nausea and vomiting. For the most part, administration of metoclopramide or alizapride prior to azacitidine is sufficient. However, in some cases nausea may be more severe and additional antiemetic medication such as a 5-HT3 antagonist may be required. Azacitidine can be administered subcutaneously or by intravenous route, although it is licensed in Europe for subcutaneous application only. The starting dose of 75 $\mathrm{mg} / \mathrm{m}^{2}$ is recommended regardless of baseline blood values. Since the 7-day regimen can be cumbersome in the outpatient setting, alternative schedules, either skipping weekends (AZA-5-2-2) or administering azacitidine for only 5 days (AZA-5) have been proposed. Recently published data from an open-label randomized phase II trial have demonstrated equal efficiency of these two schedules with the established 7-day schedule, ${ }^{63}$ although overall survival was not a study endpoint. On a cautionary note however, the majority of patients $(63 \%)$ included in this trial had lower-risk MDS. The use of the AZA-5 schedule results in a $30 \%$ dose reduction compared to the AZA-7 schedule. Though toxicity in terms of grade 3-4 hematologic adverse events was lower using the AZA-5 than the AZA-5-2-2 regimen, ${ }^{63}$ it is conceivable that a decreased dose may also translate into reduced efficacy in higher-risk MDS patients. Therefore, the use of the AZA-5 schedule cannot be routinely recommended in the higher-risk setting. For patients with lower-risk MDS and transfusion dependence who are candidates for azacitidine, the AZA-5 regimen is an appropriate choice based on its superior tolerability. ${ }^{63}$

Due to its mode of action of promoting demethylation in newly synthesized DNA, several rounds of DNA replication are necessary before azacitidine can elicit its full clinical effect. ${ }^{64-66}$ In the CALGB 9221 trial, the median number of cycles from the first treatment with azacitidine to response was 3 cycles (range, 1 to 17 cycles). ${ }^{47}$ Although $75 \%$ of responding patients achieved a response by cycle 4 , one-fourth of responding patients showed an effect as late as cycle 17 . The majority of responding patients (90\%) achieved a response by the sixth cycle. On average, best response was observed 2 cycles after initial response. Similarly, in the AZA-001 trial the median number of cycles until first response was 3 , with $50 \%$ of responding patients showing an effect after 2 courses and $87 \%$ of patients achieving a response by cycle six. ${ }^{67}$ Best response was achieved after a median of 4 cycles. On the basis of these results, it becomes evident that terminating therapy if no response is seen after a few cycles would be premature and prevent many patients from benefiting from azacitidine treatment. There is an

Table 4 Common side effects of azacitidine and their management

\begin{tabular}{|c|c|c|}
\hline Side effect & Management & Medication \\
\hline $\begin{array}{l}\text { Hematologic (neutropenia, } \\
\text { thrombocytopenia, anemia) }\end{array}$ & $\begin{array}{l}\text { Routine monitoring of } \mathrm{CBC} \\
\text { Delay administration of next cycle until } \\
\text { hematologic recovery } \\
\text { Dose reduction if necessary }\end{array}$ & $\begin{array}{l}\text { Transfusions (RBC and platelets), } \\
\text { consider G-CSF, consider prophylactic antibiotics } \\
\text { (ie, quinolones) }\end{array}$ \\
\hline Infections & $\begin{array}{l}\text { Monitor clinically for signs of infection } \\
\text { Consider prophylaxis in neutropenic pts }\end{array}$ & $\begin{array}{l}\text { Prompt initiation of antibiotic therapy, } \\
\text { consider G-CSF }\end{array}$ \\
\hline Nausea, vomiting & Symptomatic & $\begin{array}{l}\text { Alizapride or metoclopramide } 5 \text {-HT3-antagonist } \\
\text { if more severe nausea present }\end{array}$ \\
\hline Diarrhea & Symptomatic & Fluid substitution, loperamide \\
\hline Constipation & Symptomatic & Laxatives \\
\hline Injection site reaction & Symptomatic & Cool compresses, antihistamines, steroids \\
\hline
\end{tabular}

Abbreviations: $C B C$, complete blood count; RBC, red blood cells; G-CSF, granulocyte-colony stimulating factor. 
emerging consensus to treat MDS patients with azacitidine for a minimum of 6 cycles before evaluating response unless overt progression to AML or unacceptable toxicity occurs.

Another presently unresolved issue is the optimal duration of azacitidine therapy. In the CALGB 9221 trial, the range of cycles was 1 to 17 but a median cycle number was not stated. ${ }^{46}$ Best response lasted for a median of 5 cycles after achievement of first response. ${ }^{47}$ In the AZA-001 study, cycle range was 1 to 39 with a median of 9 cycles. ${ }^{48}$ Continuation of azacitidine therapy led to a higher IWG response category in $43 \%$ of responding patients and these patients received a median of 8 additional azacitidine courses. ${ }^{67}$ The median duration of hematological response (CR, PR or any hematologic improvement) in the AZA-001 trial was 13.6 months. In a small retrospective study, the outcome of patients with higher-risk MDS treated with a limited number of azacitidine cycles was analyzed. ${ }^{6}$ Patients were treated for a median of 4 cycles, with only 2 additional cycles administered as consolidation if a response was achieved after 4 cycles. Although patients clearly benefited even from this limited number of treatment courses, remission rates were lower and median survival was inferior to the AZA-001 trial where treatment was continued until progression.

The distinct mechanism of action of 5-azacitidine infers continuing benefit with prolonged therapy, and indeed this seems to be borne out by the results of the two randomized phase III trials. However, the optimal duration of therapy has not been clearly defined. In the absence of unacceptable toxicity, continued azacitidine treatment seems appropriate in responding patients and may maximize patient benefit. Of note, achievement of complete remission is not a prerequisite for attaining a survival benefit with azacitidine in higher-risk MDS. As further analyses of patient subgroups in the AZA-001 trial have shown, even hematologic improvement as defined by the IWG criteria confers an overall survival advantage for patients treated with azacitidine. ${ }^{69}$ In this analysis, one year survival was improved with azacitidine compared to conventional care in all IWG response categories. These results call into question the current paradigm that achievement of complete remission is necessary to prolong survival in MDS. ${ }^{50}$

\section{Relapse following azacitidine treatment}

A frequent problem for MDS patients treated with azacitidine is relapse or disease progression some time after discontinuation of therapy. At this time, there are insufficient data to draw definite conclusions on re-treating patients with azacitidine at relapse. However, anecdotal reports suggest this is feasible, although responses to renewed treatment seem to be inferior in terms of both quality of remission and duration of response. $^{70}$

\section{Patient-focused outcomes}

In the randomized CALGB 9221 and AZA-001 trials, patientfocused outcomes such as quality of life were independently assessed. A significantly improved quality of life for those MDS patients treated with 5-azacitidine as compared to conventional care was documented for both trials. ${ }^{71,72}$ This was evidenced by the reduction in transfusion requirements, decreased rate of infections and hospitalizations as well as improvement of fatigue, dyspnea, and physical functioning in patients receiving azacitidine. In our own experience, patients may have a temporary increase in fatigue during the first 2 cycles of therapy. However, as azacitidine exerts its effect, fatigue and asthenia decrease and patients report improved physical functioning and sense of well-being with continued therapy. Azacitidine is generally well-tolerated with few side effects. Therefore, patients' acceptance of this treatment is usually high. However, continuation of azacitidine treatment may become an issue for patients after a prolonged period of therapy, especially if only stable disease has been achieved and treatment success is not obvious. The decision to continue azacitidine therapy in patients with stable disease should be at the treating physician's discretion and take into account whether or not the patient is motivated to continue therapy. Patients who achieve hematologic improvement, PR or CR with acceptable toxicity under azacitidine therapy should be urged to continue treatment to maximize their benefit.

\section{Conclusions}

The approval of the demethylating agents azacitidine and decitabine for the treatment of MDS with higher-risk disease represents a significant advance for this group of patients, for whom treatment options have been very limited in the past. Approximately 50\% of higher-risk MDS patients can be expected to respond to azacitidine with improvement of peripheral blood values, reduction in transfusion requirement and increased quality of life. Moreover, time to progression to AML is prolonged and overall survival significantly increased by treatment with azacitidine. Side effects are generally predictable and manageable by simple prophylactic measures. Based on these favorable clinical trial results, epigenetic therapy with azacitidine can be regarded as the primary therapy of choice for patients with higher-risk MDS 
not eligible for allogeneic SCT. In patients with lower-risk MDS, azacitidine has also produced significant response rates with achievement of transfusion independence in over $50 \%$ of cases. Therefore, azacitidine is an attractive additional therapeutic option for patients with lower-risk MDS who are transfusion dependent and have failed other therapies. Future applications include using azacitidine as a bridge to allogeneic stem cell transplantation as well as for post-transplant relapses and combining azacitidine with other novel drugs to further improve outcomes in patients with MDS.

\section{Disclosures}

KSG has served as an advisory board member for Celgene and has received support for lectures from Celgene and Novartis. CMT and CP have no potential conflicts of interest to disclose.

\section{References}

1. Williamson PJ, Kruger AR, Reynolds PJ, Hamblin TJ, Oscier DG. Establishing the incidence of myelodysplastic syndrome. Br J Haematol. 1994;87:743-745.

2. Germing U, Aul C, Niemeyer CM, Haas R, Bennett JM. Epidemiology, classification and prognosis of adults and children with myelodysplastic syndromes. Ann Hematol. 2008;87:691-699.

3. Rollison DE, Howlader N, Smith MT, et al. Epidemiology of myelodysplastic syndromes and chronic myeloproliferative disorders in the United States, 2001-2004, using data from the NAACCR and SEER programs. Blood. 2008;112:45-52.

4. Greenberg P, Cox C, LeBeau MM, et al. International scoring system for evaluating prognosis in myelodysplastic syndromes. Blood. 1997;89:2079-2088.

5. Germing U, Hildebrandt B, Pfeilstocker M, et al. Refinement of the international prognostic scoring system (IPSS) by including LDH as an additional prognostic variable to improve risk assessment in patients with primary myelodysplastic syndromes (MDS). Leukemia. 2005;19:2223-2231.

6. Malcovati L, Germing U, Kuendgen A, et al. Time-dependent prognostic scoring system for predicting survival and leukemic evolution in myelodysplastic syndromes. J Clin Oncol. 2007;25: 3503-3510.

7. Park MJ, Kim HJ, Kim SH, et al. Is International Prognostic Scoring System (IPSS) still standard in predicting prognosis in patients with myelodysplastic syndrome? External validation of the WHO Classification-Based Prognostic Scoring System (WPSS) and comparison with IPSS. Eur J Haematol. 2008;81:364-373.

8. Hellström-Lindberg E, Negrin R, Stein R, et al. Erythroid response to treatment with G-CSF plus erythropoietin for the anaemia of patients with myelodysplastic syndromes: proposal for a predictive model. $\mathrm{Br} J$ Haematol. 1997;99:344-351.

9. Jädersten M, Montgomery SM, Dybedal I, Porwit-MacDonald A, Hellström-Lindberg E. Long-term outcome of treatment of anemia in MDS with erythropoietin and G-CSF. Blood. 2005;106: 803-811.

10. List A, Kurtin S, Roe DJ, et al. Efficacy of lenalidomide in myelodysplastic syndromes. N Engl J Med. 2005;352:549-557.

11. List A, Dewald G, Bennett J, et al. Lenalidomide in the myelodysplastic syndrome with chromosome 5q deletion. $N$ Engl J Med. 2006;355: 1456-1465.
12. Raza A, Reeves JA, Feldman EJ, et al. Phase 2 study of lenalidomide in transfusion-dependent, low-risk, and intermediate-1 risk myelodysplastic syndromes with karyotypes other than deletion 5q. Blood. 2008;111:86-93.

13. Lim ZY, Killick S, Germing U, et al. Low IPSS score and bone marrow hypocellularity in MDS patients predict hematological responses to antithymocyte globulin. Leukemia. 2007;21:1436-1441.

14. Saunthararajah Y, Nakamura R, Wesley R, Wang QJ, Barrett AJ. A simple method to predict response to immunosuppressive therapy in patients with myelodysplastic syndrome. Blood. 2003;102: 3025-3027.

15. Park S, Grabar S, Kelaidi C, et al. Predictive factors of response and survival in myelodysplastic syndrome treated with erythropoietin and G-CSF: the GFM experience. Blood. 2008;111:574-582.

16. Jädersten M, Malcovati L, Dybedal I, et al. Erythropoietin and granulocyte-colony stimulating factor treatment associated with improved survival in myelodysplastic syndrome. J Clin Oncol. 2008; 26:3607-3613.

17. de Witte T, Suciu S, Verhoef G, et al. Intensive chemotherapy followed by allogeneic or autologous stem cell transplantation for patients with myelodysplastic syndromes (MDSs) and acute myeloid leukemia following MDS. Blood. 2001;98:2326-2331.

18. Kroger N, Bornhauser M, Ehninger G, et al. Allogeneic stem cell transplantation after a fludarabine/busulfan-based reduced-intensity conditioning in patients with myelodysplastic syndrome or secondary acute myeloid leukemia. Ann Hematol. 2003;82:336-342.

19. Ho AY, Pagliuca A, Kenyon M, et al. Reduced-intensity allogeneic hematopoietic stem cell transplantation for myelodysplastic syndrome and acute myeloid leukemia with multilineage dysplasia using fludarabine, busulphan, and alemtuzumab (FBC) conditioning. Blood. 2004;104:1616-1623.

20. Nachtkamp K, Kundgen A, Strupp C, et al. Impact on survival of different treatments for myelodysplastic syndromes (MDS). Leuk Res. 2009;33:1024-1028.

21. Appelbaum FR, Anderson J. Allogeneic bone marrow transplantation for myelodysplastic syndrome: outcomes analysis according to IPSS score. Leukemia. 1998;12 Suppl 1:S25-S29.

22. Platzbecker U, Bornhauser M, Germing U, et al. Red blood cell transfusion dependence and outcome after allogeneic peripheral blood stem cell transplantation in patients with de novo myelodysplastic syndrome (MDS). Biol Blood Marrow Transplant. 2008;14:1217-1225.

23. Sorror ML, Sandmaier BM, Storer BE, et al. Comorbidity and disease status based risk stratification of outcomes among patients with acute myeloid leukemia or myelodysplasia receiving allogeneic hematopoietic cell transplantation. J Clin Oncol. 2007;25:4246-4254.

24. Hallemeier CL, Girgis MD, Blum WG, et al. Long-term remissions in patients with myelodysplastic syndrome and secondary acute myelogenous leukemia undergoing allogeneic transplantation following a reduced intensity conditioning regimen of 550 cGy total body irradiation and cyclophosphamide. Biol Blood Marrow Transplant. 2006;12:749-757.

25. de Lima M, Anagnostopoulos A, Munsell M, et al. Nonablative versus reduced-intensity conditioning regimens in the treatment of acute myeloid leukemia and high-risk myelodysplastic syndrome: dose is relevant for long-term disease control after allogeneic hematopoietic stem cell transplantation. Blood. 2004;104:865-872.

26. Deeg HJ, Guardiola P. Allogeneic hemopoietic stem cell transplantation in patients with myelodysplastic syndrome or myelofibrosis. Int $J$ Hematol. 2002;76 Suppl 2:29-34.

27. Wallen H, Gooley TA, Deeg HJ, et al. Ablative allogeneic hematopoietic cell transplantation in adults 60 years of age and older. J Clin Oncol. 2005;23:3439-3446.

28. Esteller M. Profiling aberrant DNA methylation in hematologic neoplasms: a view from the tip of the iceberg. Clin Immunol. 2003;109: $80-88$.

29. Jones PA, Baylin SB. The fundamental role of epigenetic events in cancer. Nat Rev Genet. 2002;3:415-428. 
30. Robertson KD. DNA methylation and human disease. Nat Rev Genet 2005;6:597-610.

31. Aoki E, Uchida T, Ohashi H, et al. Methylation status of the p15INK4B gene in hematopoietic progenitors and peripheral blood cells in myelodysplastic syndromes. Leukemia. 2000;14:586-593.

32. Aggerholm A, Holm MS, Guldberg P, Olesen LH, Hokland P. Promoter hypermethylation of p15INK4B, HIC1, CDH1, and ER is frequent in myelodysplastic syndrome and predicts poor prognosis in early-stage patients. Eur J Haematol. 2006;76:23-32.

33. Christiansen DH, Andersen MK, Pedersen-Bjergaard J. Methylation of p15INK4B is common, is associated with deletion of genes on chromosome arm $7 \mathrm{q}$ and predicts a poor prognosis in therapy-related myelodysplasia and acute myeloid leukemia. Leukemia. 2003;17:1813-1819.

34. Quesnel B, Guillerm G, Vereecque R, et al. Methylation of the p15(INK4b) gene in myelodysplastic syndromes is frequent and acquired during disease progression. Blood. 1998;91:2985-2990.

35. Tien HF, Tang JH, Tsay W, et al. Methylation of the p15(INK4B) gene in myelodysplastic syndrome: it can be detected early at diagnosis or during disease progression and is highly associated with leukaemic transformation. Br J Haematol. 2001;112:148-154.

36. Grovdal M, Khan R, Aggerholm A, et al. Negative effect of DNA hypermethylation on the outcome of intensive chemotherapy in older patients with high-risk myelodysplastic syndromes and acute myeloid leukemia following myelodysplastic syndrome. Clin Cancer Res. 2007;13:7107-7112.

37. Jones PA, Taylor SM, Wilson VL. Inhibition of DNA methylation by 5-azacytidine. Recent Results Cancer Res. 1983;84:202-211.

38. Daskalakis M, Nguyen TT, Nguyen C, et al. Demethylation of a hypermethylated P15/INK4B gene in patients with myelodysplastic syndrome by 5-Aza-2'-deoxycytidine (decitabine) treatment. Blood. 2002;100:2957-2964.

39. Raj K, John A, Ho A, et al. CDKN2B methylation status and isolated chromosome 7 abnormalities predict responses to treatment with 5-azacytidine. Leukemia. 2007;21:1937-1944.

40. Kaminskas E, Farrell A, Abraham S, et al. Approval summary: azacitidine for treatment of myelodysplastic syndrome subtypes. Clin Cancer Res. 2005;11:3604-3608.

41. Glover AB, Leyland-Jones B. Biochemistry of azacitidine: a review. Cancer Treat Rep. 1987;71:959-964.

42. Momparler RL. Pharmacology of 5-Aza-2'-deoxycytidine (decitabine). Semin Hematol. 2005;42:S9-S16.

43. Jabbour E, Issa JP, Garcia-Manero G, Kantorjian H. Evolution of decitabine development. Cancer. 2008;112:2341-2351.

44. Kaminskas E, Farrell AT, Wang YC, Sridhara R, Pazdur R. FDA drug approval summary: azacitidine (5-azacytidine, Vidaza) for injectable suspension. Oncologist. 2005;10:176-182.

45. Chabot GG, Rivard GE, Momparler RL. Plasma and cerebrospinal fluid pharmacokinetics of 5-Aza-2'-deoxycytidine in rabbits and dogs. Cancer Res. 1983;43:592-597.

46. Silverman LR, Demakos EP, Peterson BL, et al. Randomized controlled trial of azacitidine in patients with the myelodysplastic syndrome: a study of the cancer and leukemia group B. J Clin Oncol. 2002;20: 2429-2440.

47. Silverman LR, McKenzie DR, Peterson BL, et al. Further analysis of trials with azacitidine in patients with myelodysplastic syndrome: studies 8421, 8921, and 9221 by the Cancer and Leukemia Group B. J Clin Oncol. 2006;24:3895-3903.

48. Fenaux P, Mufti GJ, Hellstrom-Lindberg E, et al. Efficacy of azacitidine compared with that of conventional care regimens in the treatment of higher-risk myelodysplastic syndromes: a randomised, open-label, phase III study. Lancet Oncol. 2009;10:223-232.

49. Cheson BD, Bennett JM, Kantarjian H, et al. Report of an international working group to standardize response criteria for myelodysplastic syndromes. Blood. 2000;96:3671-3674.

50. Cheson BD, Greenberg PL, Bennett JM, et al. Clinical application and proposal for modification of the International Working Group (IWG) response criteria in myelodysplasia. Blood. 2006;108:419-425.
51. Wijermans PW, Krulder JW, Huijgens PC, Neve P. Continuous infusion of low-dose 5-Aza-2'-deoxycytidine in elderly patients with high-risk myelodysplastic syndrome. Leukemia. 1997;11:1-5.

52. Wijermans P, Lubbert M, Verhoef G, et al. Low-dose 5-Aza-2'deoxycytidine, a DNA hypomethylating agent, for the treatment of high-risk myelodysplastic syndrome: a multicenter phase II study in elderly patients. J Clin Oncol. 2000;18:956-962.

53. Kantarjian H, Issa JP, Rosenfeld CS, et al. Decitabine improves patient outcomes in myelodysplastic syndromes: results of a phase III randomized study. Cancer. 2006;106:1794-1803.

54. Steensma DP, Baer MR, Slack JL, et al. Multicenter study of decitabine administered daily for 5 days every 4 weeks to adults with myelodysplastic syndromes: the alternative dosing for outpatient treatment (ADOPT) trial. J Clin Oncol. 2009;27:3842-3848.

55. Wijermans P SS, Baila L, Platzbecker U, et al. Low dose decitabine versus best supportive care in elderly patients with intermediate or high risk MDS not eligible forintensive chemotherapy: Final results of the randomized phase III study (06011) of the EORTC leukemia and German MDS study groups. Blood. 2008;112:Abstr 226.

56. Lemaire M, Chabot GG, Raynal NJ, et al. Importance of dose-schedule of 5-aza-2'-deoxycytidine for epigenetic therapy of cancer. $B M C$ Cancer. 2008;8:128.

57. Haase D, Germing U, Schanz J, et al. New insights into the prognostic impact of the karyotype in MDS and correlation with subtypes: evidence from a core dataset of 2124 patients. Blood. 2007;110:4385-4395.

58. Knipp S, Hildebrand B, Kundgen A, et al. Intensive chemotherapy is not recommended for patients aged $>60$ years who have myelodysplastic syndromes or acute myeloid leukemia with high-risk karyotypes. Cancer. 2007;110:345-352.

59. Mufti GJ, Garcia-Manero G, Hovarth N, Lim Z, et al. Prolonged survival in higher-risk myelodysplastic syndrome (MDS) patients with $-7 / \operatorname{Del}(7 q)$ treated with azacitidine (AZA). Haematologica. 2008;93:Abstr 0928.

60. Mufti GJ, Fenaux P, Hellström-Lindberg E, Santini V, et al. Treatment of high-risk MDS patients (pts) with $-7 / \operatorname{del}(7 q)$ with azacitidine (AZA) versus conventional care regimens (CCR): Effects on overall survival (OS). J Clin Oncol. 2008;26:Abstr 7033.

61. Ruter B, Wijermans P, Claus R, Kunzmann R, Lubbert M. Preferential cytogenetic response to continuous intravenous low-dose decitabine (DAC) administration in myelodysplastic syndrome with monosomy 7 . Blood. 2007;110:1080-1082; Author reply 1083.

62. Seymour JF, Fenaux P, Silverman LB, et al. Effects of azacitidine (AZA) vs conventional care regimens (CCR) in elderly ( $>=75$ years) patients (pts) with myelodysplastic syndromes (MDS) from the AZA-001 survival trial. Blood. 2008;112:3629a.

63. Lyons RM, Cosgriff TM, Modi SS, et al. Hematologic response to three alternative dosing schedules of azacitidine in patients with myelodysplastic syndromes. J Clin Oncol. 2009;27:1850-1856.

64. Robertson KD. DNA methylation, methyltransferases, and cancer. Oncogene. 2001;20:3139-3155.

65. Christman JK. 5-Azacytidine and 5-aza-2'-deoxycytidine as inhibitors of DNA methylation: mechanistic studies and their implications for cancer therapy. Oncogene. 2002;21:5483-5495.

66. Stresemann C, Lyko F. Modes of action of the DNA methyltransferase inhibitors azacytidine and decitabine. Int J Cancer. 2008;123:8-13.

67. Silverman LR, Fenaux P, Mufti GJ, et al. The effects of continued azacitidine (AZA) treatment cycles on response in higher-risk patients (pts) with myelodysplastic syndromes (MDS). Blood. 2008;112:227a.

68. Müller-Thomas C, Schuster T, Peschel C, Götze KS. A limited number of 5-azacitidine cycles can be effective treatment in MDS. Ann Hematol. 2009;88:213-219.

69. List AF, Fenaux P, Mufti GJ, Hellström-Lindberg E, et al. Effect of azacitidine (AZA) on overall suvival in higher-risk myelodysplastic syndromes (MDS) without complete remission. J Clin Oncol. 2008; 26:Abstr 7006

70. Raj K, Mufti GJ. Azacytidine (Vidaza(R)) in the treatment of myelodysplastic syndromes. Ther Clin Risk Manag. 2006;2:377-388. 
71. Kornblith AB, Herndon JE 2nd, Silverman LR, et al. Impact of azacytidine on the quality of life of patients with myelodysplastic syndrome treated in a randomized phase III trial: a Cancer and Leukemia Group B study. J Clin Oncol. 2002;20:2441-2452.
72. Santini V FP, Mufti GJ, Hellström-Lindberg E, et al. Patient outcome during prolonged survival in patients (Pts) with high-risk myelodysplastic syndromes (MDS) treated with azacitidine (AZA). J Clin Oncol. 2008;26:Abstr 7028.

\section{Publish your work in this journal}

Cancer Management and Research is an international, peer-reviewed open access journal focusing on cancer research and the optimal use of preventative and integrated treatment interventions to achieve improved outcomes, enhanced survival and quality of life for the cancer patient. The journal welcomes original research, clinical \& epidemiological studies, reviews \& evaluations, guidelines, expert opinion \& commentary, case reports \& extended reports. The manuscript management system is completely online and includes a very quick and fair peerreview system, which is all easy to use. Visit http://www.dovepress.com/ testimonials.php to read real quotes from published authors. 